\title{
Psychologie masculine et PMA
}

\author{
S. Mimoun \\ "Centre d'Andrologie et de la Reproduction", \\ Service d'Urologie de l'Hôpital Cochin (Pr B. Debré), Paris.
}

\section{RESUME}

Les P.M.A. avec leurs aspects rationnellement magiques sont assez souvent souhaitées par la femme. L'homme, par contre, est plus attentiste, tout au moins au début.

Le narcissisme masculin est d'abord atteint par l'inaptitude sexuelle ; l'impact traumatique est particulier au cas par cas, il n'est pas proportionnel à la "gravité" de l'infertilité.

Enfin, notons que la découverte de l'infertilité est toujours un traumatisme psychologique pour l'homme et les conséquences peuvent être multiples : état dépressif, culpabilité, abandon, troubles sexuels, sexualité extra conjugale, hyperactivité professionnelle, etc ... bref, une crise d'identité.

Pour harrison et coll. [3] l'infertilité est une des situations les plus stressantes qu'un couple en tant que couple puisse rencontrer.

Notre rôle de praticien est bien sûr de prendre acte de ces différents paramètres et d'aider l'homme (et la femme) à passer ce cap et à dépasser ce qu'ils vivent comme un drame. C'est alors que l'on pourra se servir des P.M.A. comme d'un outil réaliste qui les accompagne et non pas les considérer comme un moyen magique qui les exclut.
Mots-clés : P.M.A., psychologie, andrologie, sexualité.

Quand un couple est infertile, la femme est prête, la plupart du temps, elle est même "pressée" de s'engager dans le soutien médical de sa demande d'enfant. Les PMA avec leur aspects rationnellement magiques sont donc assez souvent souhaitées.

L'homme, par contre, est plus attentiste tout au moins au début.

En apparence, le désir d'enfant et la lutte contre le temps (ce qui donne un climat d'urgence à ces consultations) est beaucoup plus manifeste chez les femmes.

En général, l'homme accompagne, suit facilement, ou au contraire résiste à la demande de sa femme, mais il la précède rarement. Peut-être parce qu'il ne sent pas le compte à rebours imposé par l'horloge biologique (de la périménopause chez la femme), mais ce n'est sûrement pas la seule explication.

Dans la lutte contre l'infertilité conjugale, l'homme (comme la femme) traverse une épreuve psychologique plus ou moins marquée. Nous savons bien que l'impact traumatique n'est pas proportionnel à la "gravité" de l'infertilité, mais les situations sont quelquefois différentes si la PMA est faite dans le cadre d'une infertilité exclusivement masculine, à fortiori, s'il s'agit d'une azoospermie et de l'utilisation de PMA avec le sperme d'un donneur. Si l'infertilité conju- 
gale est inexpliquée, le contexte est aussi différent. Pour que l'exposé soit plus clair, nous allons voir successivement les circonstances cliniques, les aspects psychologiques, la sexualité et la problématique conjugale.

\section{LES CIRCONSTANCES CLINIQUES}

Au cours des PMA, n'oublions pas que la grande majorité des soins se fait au niveau de la femme, il peut donc y avoir désinvestissement du mari. L'homme peut avoir le sentiment d'être exclu de la relation médecin-patiente [6]. Il est capital de l'associer au maximum dans les démarches et de restituer les deux partenaires en position de demandeur, si l'on veut aider au maintien du désir libidinal.

Par exemple, soulignons une notion élémentaire très importante à nos yeux. Lors d'un bilan d'infertilité féminine qui va faire appel aux PMA, n'oublions pas l'examen clinique de l'homme. Cet examen est essentiel. Non seulement pour la recherche d'anomalies masculines associées : atrophie testiculaire, varicocèle etc... mais aussi et surtout même, pour rassurer cet homme sur sa normalité génitale. Il ne viendrait à l'idée d'aucun gynécologue, me semble-t-il, de prescrire une hystérographie ou des dosages hormonaux à une femme avant même de l'avoir examinée, c'est pourtant ce qui est fait quand on prescrit un spermogramme d'emblée avant tout examen ; ou a fortiori en le donnant à la femme sans voir le mari.

Et même s'il a déjà eu au moins un spermogramme et sait comment il vit les contraintes du recueil. Mais qu'en sera-t-il le jour de la FIV?

L'xamen clinique est à la base du contact avec le patient. N'oublions pas aussi de commenter cet examen. L'homme est toujours rassuré quand, à la fin de la consultation, on peut lui dire que cliniquement tout est normal. C'est son appareil génital, donc sexuel, qui est qualifié de normal. Et cela est rassurant pour lui, même si l'infertilité n'est pas résolue. Les mots que l'on emploie ici pèsent de tout leur poids. Des mots comme "sperme paresseux, plutôt faible, glaires hostiles ..." peuvent renforcer la vision d'une image du corps dévalorisé ?

Le discours médical qui vise à l'efficacité et ne considère le rapport sexuel que dans son adéquation à la reproduction, impose une norme qui ne tient compte ni du désir ni du plaisir [1].

Rappelons enfin qu'en général quand un couple est fertile, il ne parle pas spécialement de la masturbation, cela fait partie de ce qui est non dit. La masturbation n'est pas un acte sexuel neutre, ainsi Czyba et coll [1] ont remarqué que $2 \%$ de leurs consultants n'arrivent pas à s'y résoudre, ce pourcentage atteignant $9 \%$ lorsque ces patients ont dépassé $40 \mathrm{ans}$.

Quand on donne une ordonnance pour le spermogramme sans aucune explication ou a fortiori quand on propose une IAC ou une FIV, on confronte cet homme avec la masturbation sans aucune préparation, et ceci il le vit rarement très bien, que ce soit visà-vis de lui-même, vis-à-vis de sa femme, ou encore vis-à-vis du personnel soignant.

Au début d'une infertilité conjugale l'homme peut avoir divers comportements classés par Béatrice Koeppel [4], il n'y a pas d'attitude masculine univoque bien sûr.

- Il peut par une attitude phobique éviter d'aborder réellement le problème. Cela peut être camouflé derrière un activisme professionnel ou sportif, mis en place pour effacer voire nier l'évènement en remettant toujours à plus tard la prise en compte de ce problème, comme s'il avait le temps.

- Il peut aussi accepter l'idée de consulter pour faire plaisir à sa femme, ou même tout simplement pour avoir la paix.

- Il peut enfin se sentir personnellement concerné. 
L'infertilité (vécue comme provisoire dans le temps), le touche peu au début, à la différence de l'inaptitude sexuelle qui atteint son narcissisme d'emblée. Plus que les femmes, les hommes ont tendance à remettre à plus tard un certain nombre de projets et de réalisations. Ils font comme s'ils étaient éternels et que le temps avait moins de prise sur eux. Leurs références temporelles essentielles sont extérieures à leurs corps.

Même quand ils parlent du temps qui passe, les hommes font souvent référence au temps matériel, extérieur, au temps perdu qui gêne leurs autres activités ... Ce n'est pas le temps intime, introjecté propre à la femme infertile.

Quand un diagnostic de stérilité incurable a été porté, le contexte est tout autre chez les hommes.

En cas d'azoospermie d'origine secrétoire par exemple, les hommes se sentent alors atteint dans leur corps viril. Ils ont l'impression qu'à cause d'eux le temps générationnel s'arrête. Ils peuvent souhaiter, refuser, voire "cacher" une demande d'IAD (insémination artificielle avec sperme de donneur) en fonction de ce qu'ils projettent de leur identité sexuelle et de la place qu'ils veulent prendre dans l'espace et dans le temps (à travers la filiation). Comme $\mathrm{Mr} \mathrm{C}$. qui nous demande si l'on ne peut faire une IAD à sa femme en lui faisant croire qu'il s'agit de son propre sperme. Entre homme on peut se comprendre, insiste-t-il. Il cherche désespérément une complicité qui puisse le maintenir dans l'illusion d'une filiation.

"Je ne peux pas supporter de ne pas avoir marqué mon temps, mon passage sur terre aura été inutile. Il n'en restera rien, même pas un enfant".

\section{LES ASPECTS PSYCHOLOGIQUES}

En clinique nous constatons que de plus en plus d'hommes sont prêts à envisager une origine psychogène masculine à leur infertilité conjugale et ce d'autant plus :

- que l'infertilité reste inexpliquée ;

- qu'il a existé dans l'enfance des traumatismes que le patient a repérés ;

- que les mots employés pour constater l'infertilité sont d'un registre qualitatif.

$\mathrm{Au}$ cours des consultations certains hommes, se posent, nous posent la question du hasard, de la dette, du désir inconscient ... Ils se demandent en fait, s'ils n'arrivent pas à avoir d'enfant, ou bien à faire un enfant à leur femme, ou encore s'ils n'arrivent pas à être père, ce qui ne soulève pas tout à fait les mêmes problèmes.

La question du désir d'enfant chez l'homme "traverse" celle de la paternité [5]. Habituellement, elle est plus désir de transmettre, plutôt que de donner une vie, ce qui est différent pour une femme.

Comme l'a bien souligné Pierre Lachcar [5], l'homme infertile peut:

- être un homme qui souffre de son infertilité ;

- ou être en souffrance comme on dit qu'une lettre ou un colis est en souffrance : il est arrêté, dans une attente ;

- mais il peut être aussi un homme en souffrance, souffrance en tant qu'homme, au sens où c'est en tant que sujet de sexe masculin qu'il est interpellé. Est-il encore un homme s'il n'a pas d'enfants ? C'est toute la question de l'identité sexuée qui se trouve posée là, au travers d'un enfant qui ne vient pas, qui ne s'inscrit pas dans la généalogie ;

- enfin, l'homme infertile est aussi en souffrance d'une reconnaissance car même s'il n'est pas reconnu lui-même comme une personne infertile, alors qu'on reconnaît infertile son sperme, on risque de déplacer la question de la paternité sur la biologie, éventuellement dans le but de protéger l'homme d'ailleurs. Or, ce ne sont 
pas des gamètes qui feront la dimension symbolique de la paternité. D'autant que comme la production spermatique n'est pas recueillie par un organe féminin, elle risque de changer de statut. Elle passe de celui d'objet vivant à celui de sécrétion sexuelle, voire de déchet, isolant de ce fait l'homme de son sperme et écartant là son désir dans la procréation [5]. Mettre le sperme, la biologie, au premier plan risque de mettre certains hommes dans une place impossible au cours de ces PMA

- par ailleurs ne pas pouvoir faire un enfant est parfois vécu comme une véritable mutilation. Des patients parlent de blessure, ou même de l'amputation d'un membre. Peut-être que pour eux cela évoque t-il la façon dont ils vivent leur pénis séparé d'eux ? Cette perte est vécue comme réelle, tant l'idée de perte d'une partie de soi est fortement perçue. Il est fréquent dans ces cas que des difficultés sexuelles apparaissent dès l'annonce de l'infertilité.

Quoi qu'il en soit la découverte de l'infertilité est toujours un traumatisme psychologique pour l'homme et les conséquences peuvent être multiples : état dépressif, culpabilité, abandon de la partenaire, troubles sexuels, sexualité extra conjugale, hyperactivité professionnelle, etc ... bref, une crise d'identité [5].

\section{LA SEXUALITE AU DECOURS DES PMA}

En tant qu'organe, l'appareil génital masculin est pratiquement sans contraintes : pas de règles, pas d'examens systématiques, pratiquement pas de contraception médicale, pas de traumatisme obstétrical. En tant que symbole, il est traditionnellement celui de la puissance et de l'autorité. L'ensemble concourt cependant à une grande fragilité et à une difficulté d'adaptation aux situations inhabituelles qu'il ne peut plus contrôler. Certes, l'homme moderne est de plus en plus conscient de la dissociation entre la fertilité et la virilité, mais il reste encore dans son inconscient des plages d'incertitudes où viennent déferler certains stress des PMA. L'homme se sent parfois blessé, mais l'exprime rarement en tant que tel.

"Sa sexualité est pourtant facilement mortifiée devant ce qu'on exige de lui. Un délai d'abstinence, un recueil par masturbation post-mictionnelle, médicalisée et aseptisée au Dakin, entouré d'un pseudoérotisme de kiosque à journaux dans l'espoir que "ça vienne plus vite". Ce n'est pas la sexualité joyeuse, c'est de l'anti-sexualité qui, comme l'a si bien souligné Philippe Granet [2], permet littéralement d'inverser la célèbre sentence de la manière suivante :

"qu'importe l'ivresse pourvu qu'on ait le flacon". C'est en fait une sexualité du handicap, un détournement palliatif pour un retour final dans la norme : la conception d'un enfant" [2].

Le langage familier dit qu'il lui a fait un enfant. Or dans les PMA est-il toujours sûr de l'avoir réellement fait ? On pense un peu facilement, nous l'avons vu, qu'étant peu médicalisé il a le beau rôle, en fait ne serait-il pas plutôt tellement marginalisé qu'il deviendrait le "parent pauvre" de cette procréation? Ne risque-t-il pas parfois de croire que c'est la médecine qui a fait un enfant à sa femme? Souvenons-nous des références mythologiques où des dieux déposaient des enfants dans des corps de femmes, il n'était guère fait allusion à leurs pères nourriciers [2].

Bien que nous ayons décrit d'une manière non exhautive les multiples difficultés qui peuvent rendre le recours aux PMA très difficiles pour l'homme, précisons que dans l'ensemble, le déroulement des traitements se passe plutôt bien. Même si des attitudes qui à première vue paraissent très sereines masquent, par pudeur ou par orgueil, un profond désarroi, il est incontestable dans 
la majorité des cas que leur apparence correspond à leur réalité.

Le couple qui avait déjà peu ou pas de rapports sexuels satisfaisants, du fait entre autre de sa focalisation obsédante sur la lutte contre la stérilité, voit le besoin de sa sexualité s'annuler complètement quand le désir ou plutôt l'exigence d'enfant, prend plus encore le devant de la scène. Dans la FIV par exemple, le corps de la femme peut devenir le terrain de l'expérience, le lieu de l'intervention technique. Il s'agit presque d'un corps morcelé et si c'est le cas, l'intimité corporelle ne peut que souffrir d'une telle situation.

L'homme quant à lui à parfois plus encore que dans l'IAC du mal à éjaculer dans ces conditions stressantes. Ici il n'y a pas qu'un seul médecin, dans la relative "intimité" du cabinet médical, qui attend son sperme ; il y a toute l'équipe hospitalière.

Précisons quand même que si l'on ne met pas au second plan la situation conjugale, les techniques de procréation peuvent permettre de maintenir ou même de retrouver le désir sexuel. En effet, la programmation de PMA n'a lieu que deux ou trois fois par an. Tous les autres mois sont "libres". Le couple peut s'aimer comme il l'entend, à son rythme, sans être obsédé par l'attente d'une grossesse à chaque rapport.

En dehors de ces cycles programmés le couple se sait stérile, il est donc libre de toute attente, d'autant qu'il sait que l'on va l'aider à avoir un enfant, ce qui le rend confiant. Petit à petit, les deux partenaires se retrouvent, ils sortent de leurs réflexes obsédants : sur la polarisation du jour de l'ovulation, ou la crainte de survenue des règles ... Ils redécouvrent la spontanéité.

\section{LA PROBLEMATIQUE CONJUGALE}

En fait, quand il s'agit d'une stérilité incurable (que ce soit chez l'homme ou chez la femme), c'est la problématique conjugale qui prend le devant de la scène. Le couple stérile devra pour survivre en tant que couple, passer l'épreuve du temps. Quand un couple apprend la nouvelle de sa stérilité il y a toujours un moment de crise d'identité. L'annonce de cette nouvelle tombe souvent comme un couperet. Sentiment d'infériorité de ne pas se sentir vraiment "femme" ou vraiment "homme", et sentiment de culpabilité envers le conjoint sont très souvent mêlés. Nous pourrions dire avec Claude Sachs-Nerson [8] que c'est comme si limaginaire érotique du couple se trouvait aseptisé pour ne pas dire stérilisé, par le rituel répétitif et contraignant des explorations. La fécondation va de pair avec la fertilité imaginaire, c'est-à-dire, avec la possibilité de fantasmer seul et à deux, de faire circuler le flux de l'imaginaire autant que celui du sperme ou des ovocytes.

Une publication de Nijs et de Demyttenaere [7] fait état d'une élévation du niveau d'anxiété dans le cadre des infertilités ; ce stress engendrant par lui même une stérilité ou aggravant une infertilité des couples subfertiles. Pour Harrison et coll [3] l'infertilité est une des situations les plus stressantes (estimées grâce à des dosages de la prolactine et des tests psychologiques) qu'un couple en tant que couple, puisse rencontrer.

Certes chacun réagit en fonction de sa personnalité et de ses mécanismes de défense. Comme on l'a vu, on assiste parfois à un désespoir dépressif ou à un activisme professionnel, sportif, associatif ... mis en place pour effacer, voire pour nier, l'événement.

Du côté du conjoint un réaménagement se fait. Il y a quelquefois une surprotection de la personne stérile, considérée comme malade, mais d'autres fois il y a un conflit, la personne fertile se sentant coincée entre son désir d'enfant et la culpabilité de ce projet devant l'impossibilité de le réaliser avec son conjoint.

Tout ceci dépend non seulement du code psychologique propre à chaque individu, 
mais aussi du contexte culturel dans lequel baigne cet individu. L'homme qui vit dans un milieu où la puissance et la virité se mesurent au nombre d'enfants risque bien sûr de très mal vivre sa propre stérilité et cherchera sans doute un certain nombre de subterfuges. Tous ces éléments interviennent sur la sexualité conjugale, parfois longtemps après l'annonce de la stérilité et quelquefois même si le couple se sépare.

Quand il apprend qu'il est azoospermique, Mr G. a un air apparemment détaché. Il accepte facilement, semble-t-il, que sa femme fasse une insémination artificielle avec sperme de donneur (IAD) puis une FIV. Rapidement il ne se sent plus concerné. Il quitte sa femme peu de temps après, et se lance dans de nombreuses aventures "amoureuses". Cette période don juanesque cesse cependant brusquement à la suite d'une "panne" sexuelle. La première chose qu'il dit quand il revient consulter, deux ans après, c'est : "je ne suis plus un homme, ce n'est pas étonnant avec le sperme que j'ai".

De fait, malgré les apparences il n'a rien oublié de son bilan.

Le couple stérile devra survivre en tant que couple, passer cette épreuve d'identité conjugale et trouver un nouveau but à sa vie.

Sentiments de culpabilité envers le conjoint et sentiments d'infériorité, s'entremêlent sans cesse.

Il n'est pas rare que le couple stérile se retrouve seul, face à cette crise d'identité, surtout dans les grandes villes où chacun est isolé et où il n'y a personne dans l'entourage pour le soutenir. Dans ce cas, il peut avoir tendance à se replier sur luimême et à se couper un peu plus des autres, espérant éviter de la sorte les allusions sur cet enfant qui ne vient pas, ou éviter la souffrance réveillée par les naissances dans l'entourage ...

A cette période, le couple peut se défaire. L'homme (ou la femme) peut rechercher une "solution" extra-conjugale. Quelquefois cependant, les deux partenaires vivent ce drame ensemble et cherchent à survivre en tant que couple après avoir retrouvé une nouvelle identité.

Le couple découvrira peut-être alors que vouloir être père ou mère, c'est d'abord se désirer ensemble pour qu'ensuite il en sorte un enfant.

\section{BIBLIOGRAPHIE}

1. CZYBA J.C., CHORIER H., CLEMENT J.L. : Nouvelles technologies de la médecine de la reproduction et sexualité. Médecine et Hygiène, 1985, 43, 1202.

2. GRANET Ph. : L'impossible place de l'homme au décours des PMA in Premières assises de la SFGOP - Paris 1990.

3. HARRISON R.F., O'MOORE R.R., O'MOORE A.M. : Stress and fertility, some modalities of investigations and treatment in couples with inexplained infertility. Int. J. fertil., 1986, 31, 153-159.

4. KOEPPEL B. : Le dilemme des hommes FIV, Gynécologie Psychosomatique $n^{\circ} 11,1994$.

5. LACHCAR P. : "L'homme infertile : un homme en souffrance", Contr. Fert. Sex., 1992, vol 20, nº 7-8, 735-736.

6. MIMOUN S., CHAUSSIN E. : In "L'Univers Masculin", ed. le Seuil, Paris 1995.

7. NIJS P., DEMYTTENAERE K. : Fertilisation in vitro et transfert d'embryon. Cahiers de sexologie clinique, 1986, 12, 72, 43-48.

8. SACHS-NERSON C. : Comment s'en passer ... Sexualité et fertilité aujourd'hui. Contr. Fert. Sex., 1987, 15, 12, 1171-1173.

\section{ABSTRACT \\ Male psychology and M.A.P.}

\section{S. Mimoun}

M.A.P. and their rationally magical aspects are often desired by the woman, rather more than by the man who would more readily follow a waitand-see policy, at least at the beginning. 
Masculine narcissism inclines to be wounded essentially by sexual deficiency and infertility lived first as a temporary problem, touch him less, at lest apparently, at the outset of the situation.

But in men as in women, we are well aware that the traumatic impact is particular and specific to each case and each individual, and never proportional to the so-called gravity of the infertility.

Furthermore let us keep in mind that in itself the discovery of the infertility is always a psychological trauma for a man, with various possible consequences i.e. depressive state, culpability, behaviour of abandon, sexual problems, extra-marital sexuality, profes- sional hyperactivity ... a.s.o., in short an identity crisis.

For Harrison and al. [3], infertility is one of the direst and most stressful situations a couple may encounter as a couple.

Our role as practitioners is of course to take note of those different parameters so as to help man, and woman, to round that cape and to get over an ordeal they consider a tragedy. Then, and then only, will we be able to make use of the M.A.P. as of a realistic instrument to accompany them with, and not as a magical mean which excludes them.

Key-words : M.A.P., psychology, andrology, sexuality. 